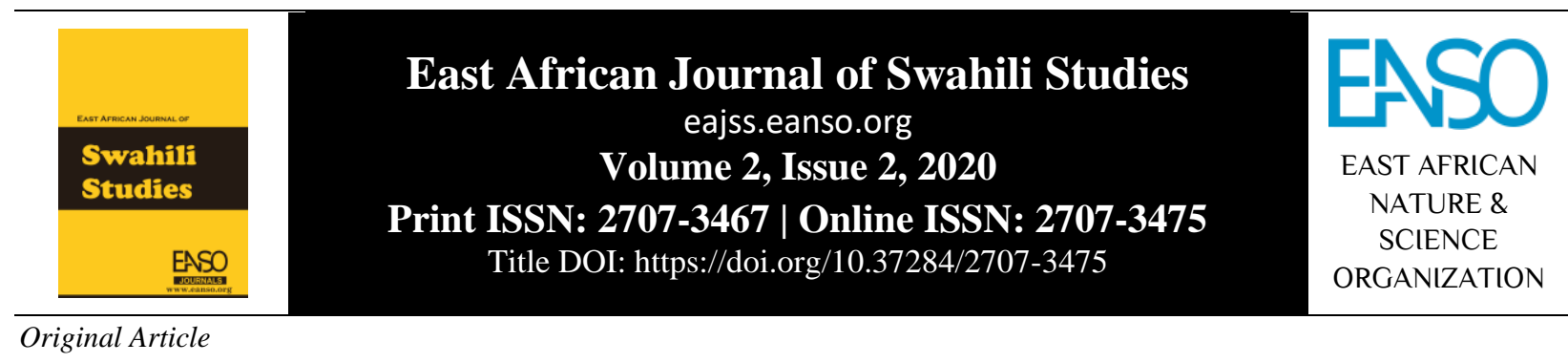

Mchango wa Tasinia ya Sheria katika Ukuzaji na Uenezaji wa Kiswahili Tanzania: Mifano kutoka Mahakama ya Tanzania na Taasisi za Wadau wa Sheria

\author{
Dr. Joseph Hokororo Ismail ${ }^{1^{*}}$ \\ ${ }^{1}$ Chuo Cha Uongozi wa Mahakama Lushoto, S.L.P. 20, Lushoto, Tanzania. \\ *Barua pepe ya mawasiliano: jihokororo@yahoo.com
}

DOI ya Nakala: https://doi.org/10.37284/eajss.2.2.160

Tarehe ya Uchapishaji: Kukua na kuenea kwa lugha ya Kiswahili nchini Tanzania kunategemea mambo mbalimbali kama vile kutumika katika maeneo na mifumo rasmi 09 Juni 2020 kwenye jamii husika. Historia ya lugha ya Kiswahili inaonekana kukua tangu wakati wa biashara ya watumwa, Waarabu, kipindi cha ukoloni wa Maneno Muhimu: Wajerumani na kipindi cha ukoloni wa Waingereza. Harakati za kusanifisha lugha ya Kiswahili kwa hatua mbalimbali za usanifishaji wake (1928-1930)

Sheria, zilichapuza kukua na kusambaa kwa lugha ya Kiswahili. Baada ya uhuru wa Wadaiwa, Tanzania, Tanganyika wakati huo, lugha ya Kiswahili ilizidi kukua hasa baada Wadaawa, ya serikali katika mwaka 1962 kuifanya kuwa lugha ya Taifa na lugha rasmi Mashauri, ya mawasiliano katika shughuli mbalimbali za kiserikali na zisizo za Shauri la Jinai, kiserikali. Vyombo mbalimbali vya kukuza Kiswahili viliundwa kama vile Shauri la Madai, Baraza la Kiswahili la Tanzania (BAKITA), Taasisi ya Ukuzaji wa Kiswahili Shauri la Mirathi. (TUKI) kutokana na kamati iliyokuwa ya usanifishaji wa lugha ya Kiswahili ya Afrika Mashariki na Idara ya Kiswahili ya Chuo Kikuu cha Dar es salaam, kuvitaja kwa uchache. Idara ya Kiswahili ya Chuo Kikuu cha Dar es Salaam ilikuwa na jukumu la kufundisha somo la Kiswahili na wataalamu wake kuandika masuala mbalimbali ya lugha ya Kiswahili. Mazoea yamekuwa kuviangalia vyombo hivi na serikali kwa ujumla kama ndio vyombo pekee vyenye wajibu wa kukuza na kuendeleza lugha ya Kiswahili Tanzania. Tukiachilia mbali vyombo hivi tulivyovitaja, yapo maeneo mengine yanayoweza kukuza na kuendeleza lugha ya Kiswahili ambayo ama hayajaangaliwa kwa kiasi cha kutosha au yamesahaulika. Moja ya maeneo ambayo matumizi ya lugha ya Kiswahili yanaweza kusukuma kukua na kuenea kwake ni eneo la tasinia ya sheria. Lengo la makala haya ni kubainisha namna tasinia ya sheria na taasisi za sheria zinavyosaidia kukua na kuenea kwa Kiswahili nchini Tanzania.

\title{
APA CITATION
}

Ismail, J. (2020). Mchango wa Tasinia ya Sheria katika Ukuzaji na Uenezaji wa Kiswahili Tanzania: Mifano kutoka Mahakama ya Tanzania na Taasisi za Wadau wa Sheria. East African Journal of Swahili Studies, 2(2), 11-22. https://doi.org/10.37284/eajss.2.2.160 


\section{CHICAGO CITATION}

Ismail, Joseph. 2020. "Mchango wa Tasinia ya Sheria katika Ukuzaji na Uenezaji wa Kiswahili Tanzania: Mifano kutoka Mahakama ya Tanzania na Taasisi za Wadau wa Sheria". East African Journal of Swahili Studies 2 (2), 11-22. https://doi.org/10.37284/eajss.2.2.160.

\section{HARVARD CITATION}

Ismail, J. (2020) "Mchango wa Tasinia ya Sheria katika Ukuzaji na Uenezaji wa Kiswahili Tanzania: Mifano kutoka Mahakama ya Tanzania na Taasisi za Wadau wa Sheria", East African Journal of Swahili Studies, 2(2), pp. 11-22. doi: 10.37284/eajss.2.2.160.

\section{IEEE CITATION}

J. Ismail, "Mchango wa Tasinia ya Sheria katika Ukuzaji na Uenezaji wa Kiswahili Tanzania: Mifano kutoka Mahakama ya Tanzania na Taasisi za Wadau wa Sheria”, EAJSS, vol. 2, no. 2, pp. 11-22, June 2020.

\section{MLA CITATION}

Ismail, Joseph. "Mchango wa Tasinia ya Sheria katika Ukuzaji na Uenezaji wa Kiswahili Tanzania: Mifano kutoka Mahakama ya Tanzania na Taasisi za Wadau wa Sheria". East African Journal of Swahili Studies, Vol. 2, no. 2, June 2020, pp. 11-22, doi:10.37284/eajss.2.2.160.

\section{UTANGULIZI}

Kiswahili ni miongoni mwa lugha kubwa zinazotumika katika mawasiliano nchini Tanzania, Afrika Mashariki na kwenye mikutano ya Umoja wa Afrika. Nchini Tanzania, Kiswahili ni lugha ya taifa iliyochangia kuleta umoja, uhuru, mshikamano na maendeleo katika nyanja mbalimbali. Kiswahili ni lugha rasmi katika shughuli za kiserikali na kijamii. Kwa kuwa si lengo la makala haya kuonesha mwelekeo wa lugha ya Kiswahili katika ukuaji na matumizi yake kikanda na kimataifa, hatutaendelea na mjadala huu na badala yake tutajiegemeza kwenye lengo la makala. Makala haya alinuia kupeleleza namna ambavyo tasinia ya sheria hasa mahakama za mwanzo, taasisi zinazofundisha sheria kwa ngazi ya astashadada, na taasisi za kijamii zinavyochangia katika kukua na kuenea kwa lugha adhimu ya Kiswahili.
Nchini Tanzania, kuna lugha mbili zinazotumika katika shughuli rasmi za kitaifa ambazo ni lugha ya Kiingereza na Kiswahili. Sheria nyingi hupitishwa kwa lugha ya Kiingereza ikisemekana kwamba bado Tanzania haina uwezo wa kuwasomesha wataalamu wa mambo ya sheria katika lugha ya Kiswahili kwa kiwango cha chuo kikuu (Mukoyogo, 1991; Rwezaura, 1993). Hata hivyo, jitihada zimefanywa na Tume ya Kurekebisha Sheria Tanzania na asasi zingine za sheria kutafsiri baadhi ya sheria zilizoandikwa kwenye lugha ya Kiingereza kwenda kwenye lugha ya Kiswahili. Lugha ya Mahakama katika ngazi ya wilaya hadi mahakama ya rufaa ni Kiingereza japo mashauri huendeshwa kwa lugha ya Kiswahili lakini nyaraka huandikwa kwa lugha ya Kiingereza isipokuwa kwa Mahakama ya Mwanzo.

\section{Jedwali 1: Lugha za Mahakama Tanzania}

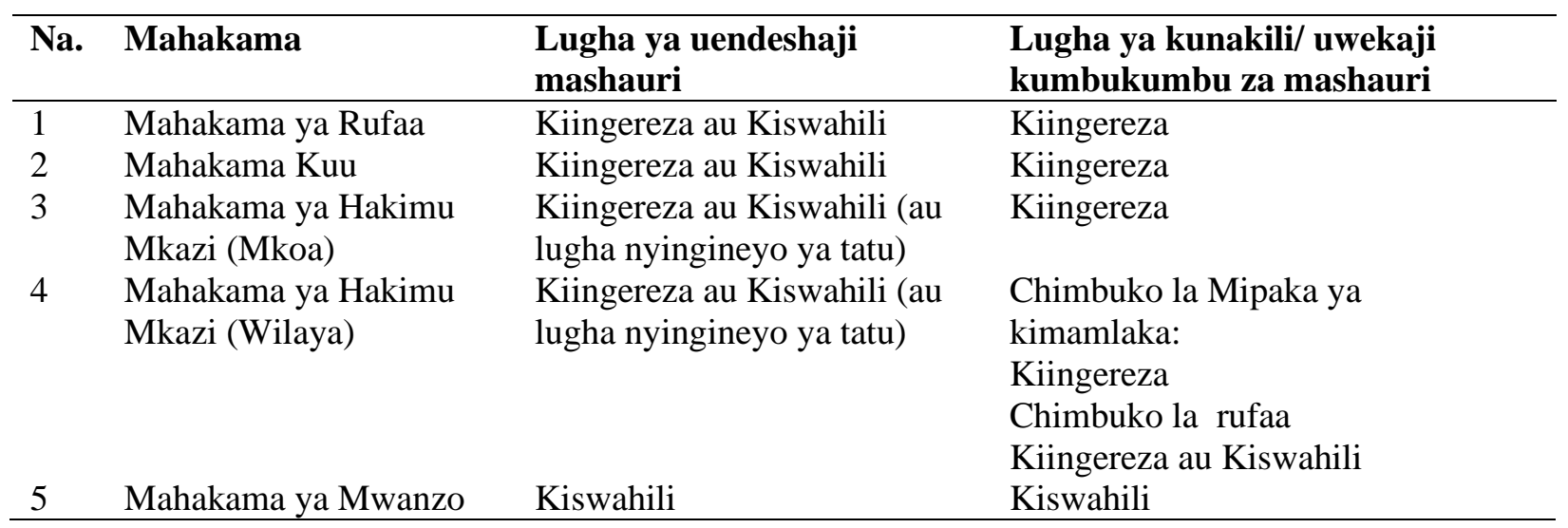


Katika Jedwali 1 tunaona kwamba kuna matumizi ya lugha ya Kiswahili yanayoweza kuchukuliwa kuwa yanaweza kuhifadhi na kukuza lugha ya Kiswahili. Maeneo hayo ni lugha ya uendeshaji mashauri na lugha ya uwekaji wa kumbukumbu za mwenendo wa mashauri. Katika lugha inayotumika kuendesha mashauri, tunaona lugha ya Kiswahili na Kiingereza hutumika katika mahakama zote isipokuwa mahakama za Mwanzo ambapo lugha ya Kiswahili hutumika. Aidha, kuna uwezekano wa matumizi ya lugha ya tatu kutegemeana na wahusika katika shauri lenyewe (Wanitzek na Twaib , 1996). Katika kunakili na kuweka kumbukumbu za mashauri, lugha ya Kiingereza hutumika isipokuwa kwenye mahakama za mwanzo ambapo lugha ya Kiswahili pekee hutumika kuhifadhi kumbukumbu. Katika Jedwali 1 tunaona pia kwamba, katika mahakama ya mwanzo, lugha ya Kiswahili hutumika kuendesha mashauri na uandikaji wa kumbukumbu ya mwenendo wa mashauri. Hii ndio sababu iliyopelekea mwandishi kuchagua mahakama za Mwanzo tu katika kuangazia mchango wa mahakama katika kukuza na kuendeleza lugha ya Kiswahili nchini Tanzania. Kifungu cha 13, kifungu kidogo cha 1 cha Sheria ya Mahakimu na Mahakama, Sura ya 11 kama ilivyofanyiwa mapitio 2002, ndicho kilichotamka kuwa katika Mahakama ya Mwanzo, lugha rasmi ya mahakama ni Kiswahili.

Makala haya yamegawanyika katika sehemu tano. Sehemu ya kwanza ni utangulizi. Sehemu ya pili inahusu njia zilizotumika katika ukusanyaji na uchakataji wa data ikifuatiwa na uwasilishaji wa matokeo. Sehemu ya tatu ni mjadala kuhusu tasinia ya sheria na uenezaji wa lugha ya Kiswahili. Sehemu ya nne inajadili changamoto na kutoa mapendekezo. Sehemu ya tano ni hitimisho.

\section{MBINU ZA UCHUNGUZI}

\section{Ukusanyaji na Uchakataji wa Data}

Makala hii ilitumia mbinu mseto katika kukusanya data. Njia mseto ilikuwa ni muhimu kwa kuwa makala imehusisha utafiti wa namna lugha ya Kiswahili inavyokuzwa katika maeneo mbalimbali yanayohusiana na masuala ya sheria. Njia hizo zinafafanuliwa kama ifuatavyo:

\section{Njia ya Ukusanyaji Data Maktabani na Makavazini}

Kulikuwa na usomaji wa machapisho anuwai. Machapisho haya yalitoka kwenye maeneo ya mahakama, kamusi za sheria, kamusi ya Kiswahili sanifu, tovuti za Tume ya kurekebisha sheria, bunge la Tanzania, na makavazi ya taasisi tatu zinazofundisha elimu ya sheria kwenye kiwango cha astashahada ya sheria. Data ilikusanywa kupitia njia mbili: njia ya kwanza ilikuwa ni ushiriki wa kuingia katika mahakama na kusikiliza uendeshwaji wa mashauri. Njia ya pili ni usomaji wa nyaraka mbalimbali zinazohusika na masuala ya mahakama kwa ujumla na mahakama ya mwanzo. Pia, mtafiti alipata fursa ya kusoma baadhi ya sheria, magazeti, vipeperushi, matangazo na matini nyingine katika ofisi za mahakama za mwanzo. Hii ilisaidia kuona lugha inayotumika kama ni Kiswahili au Kiingereza au mchanganyiko.

\section{Njia Ushiriki wa Mtafiti}

Njia hii ilihusisha kuangalia shughuli za uendeshaji wa mashauri katika mahakama za Mwanzo kwa mwezi mmoja kwa nyakati na miaka miwili tofauti wakati mwandishi alipowasimamia wanafunzi wa astashahada ya sheria walipokuwa wanafanya mazoezi kwa vitendo kwenye mahakama za mwanzo kwa siku na tarehe anuwai. Kwa kutumia njia hii mtafiti aliweza kuona jinsi mahakama zinavyoendesha mashauri hasa kuhusiana na kipengele cha matumizi ya lugha kama vile umahiri wa watumiaji wa lugha hizo, jinsi maswali wanayoulizana na majibu kati ya hakimu, wazee wa baraza, walalamikaji na washtakiwa. Mwandishi aliangalia kama matumizi ya lugha ya Kiswahili ni fasaha na sanifu au kama kuna matumizi ya lugha za kijamii au lugha ya Kiingereza. Katika njia ya pili, ambayo kimsingi ni utafiti wa maktabani, mwandishi alisoma baadhi ya sheria, magazeti, vipeperushi, matangazo na matini nyingine katika ofisi za mahakama za mwanzo. Njia hii ilisaidia kuona lugha inayotumika kama ni Kiswahili au Kiingereza au mchanganyiko.

Baada ya kukusanya data, zilichambuliwa na kuwekwa katika makundi yanayohusiana, kama vile hoja za ukuzaji wa Kiswahili zinazohusiana na mahakama na zile zinahusu taasisi nyinginezo nje 
ya mahakama na kuanza kufanya uchanganuzi wa matini mbalimbali na hatimaye kufanyiwa hitimisho kulingana na makundi husika.

\section{MCHANGO WA TASINIA YA SHERIA KATIKA UKUAJI NA UENEZAJI WA KISWAHILI}

Tuanze mjadala wetu kwa kujiuliza swali hili. Kwa nini sheria, mikataba na hukumu katika mahakama zote nchini zisitolewe ama kuandikwa kwa Kiswahili? Baadhi ya wahojiwa katika Mahakama ya Mwanzo walioulizwa kuhusu uelewa wao katika masuala ya sheria na mikataba iliyopo katika makavazi za sehemu zao za kazi hasa katika Mahakama za Mwanzo walisema hawazielewi. Hii ni kutokana na kiasi kikubwa kutokuwa na uelewa wa lugha ya Kiingereza ambapo mikataba na sheria hizo zimeandikwa kwa lugha hiyo. Ikumbukwe kwamba waathirika wa sheria na mikataba kwa lugha ngeni isiyofahamika na Watanzania wengi ni Watanzania wanaozungumza na kuwasiliana kwa lugha ya Kiswahili katika shughuli zao za kila siku. Hivyo, ipo haja kwa mamlaka zinazohusika na sheria Tanzania kuhakikisha sheria zinazotumika nchini zilizopo katika lugha ya Kiingereza zinatafsiriwa kwa lugha ya Kiswahili na hivyo sheria iandikwe kwenye lugha mbili, lugha ya Kiingereza na Kiswahili. Ingawa changamoto yaweza kuwa usambaaji wa sheria hizo kuwafikia kundi kubwa la wazungumzaji wa Kiswahili, lakini kwa kuwa na tafsiri kwa lugha ya Kiswahili ni hatua kubwa ya kukuza na kuendeleza lugha ya Kiswahili Tanzania. Kwa kufanya hivyo, sio tu Kiswahili kitaendelea na kuendelezwa, lakini pia lugha ya Kiswahili itachangia katika mawasiliano sehemu za mahakama ambapo Watanzania watapata huduma za Kimahakama kwa lugha wanayoifahamu zaidi.

\section{Jedwali 2L Lugha inayotumika katika uandishi wa sheria kimchakato}

\begin{tabular}{lll}
\hline Na. & $\begin{array}{l}\text { Hatua katika mchakato wa kutunga } \\
\text { sheria }\end{array}$ & Lugha inayotumika \\
\hline 1 & Uandishi wa sheria mama (miswada) & $\begin{array}{l}\text { Kiingereza (kukiwa na lugha ya Kiswahili ) Isipokuwa: } \\
\text { Katiba (Kiswahili) } \\
\text { Kiswahili au Kiingereza }\end{array}$ \\
3 & $\begin{array}{l}\text { Uandishi wa sheria nyinginezo } \\
\text { Majadiliano ya miswada bungeni }\end{array}$ & $\begin{array}{l}\text { Kiswahili } \\
\text { Upitishaji wa sheria mama (kanuni, } \\
\text { katiba) }\end{array}$ \\
$\begin{array}{l}\text { Utangazwaji wa Kanuni (matangazoreza, Isipokuwa Katiba (Kiswahili) } \\
\text { ya serikali) } \\
\text { Tafsiri za Sheria mama }\end{array}$ & Kiswahili au Kiingereza \\
& $\begin{array}{l}\text { Kutoka lugha ya Kingereza kwenda Kiswahili: } \\
\text { eg. penal code, Magistrate's Court Act 1963 (now } \\
\text { repealed),law of marriage Act, Ward tribunal Act } \\
\text { Kutoka lugha ya Kiswahili kwenda lugha ya Kiingereza }\end{array}$ \\
\hline
\end{tabular}

Tukirejea kwenye Jedwali la 2, tunaona kwamba lugha ya Kiswahili hutumika katika hatua mbalimbali za utungaji wa sheria kutoka kiwango cha mapendekezo, majadiliano bungeni, muswada, na hatua za kuifanya kuwa sheria. Hata pale ambapo lugha ya Kiingereza hutumika kutunga sheria ndogo, rasimu hiyo imetafsiriwa pia kwenye lugha ya Kiswahili. Kwa kufanya hivyo, lugha ya Kiswahili inakua inahifadhiwa na inasambaa kwa njia ya majadiliano yanayoendelea wakati wa kujadili miswada hiyo (Vlachopoulos, 2004).

\section{Lugha ya Uendeshaji wa Mashauri}

Matumizi ya lugha ya Kiswahili katika mahakama za Tanzania inaonekana kusaidia kukua na kuenea kwa lugha ya Kiswahili. Jambo hili halifanyiki kwa makusudio hayo bali wakati wa kutekeleza majukumu ya kisheria ndipo lugha ya Kiswahili inaenea na kukua. Lugha ya Kiswahili hutumika katika kuendesha mashauri. Wakati wa kuendesha mashauri, kumbukumbu ya mwenendo wa shauri huwekwa kwa Kiswahili. Tukiangazia zaidi katika tasinia hii ya sheria tunaweza pia kujiuliza swali la msingi kuhusu wafasiri wa sheria husika mahakamani. Swali la msingi ni je, nchini Tanzania 
viongozi wanaotafsiri sheria, majaji na mahakimu ni Waswahili au sio Waswahili? Majibu ya swali hili sio rahisi isipokuwa upo ukweli kwamba kuna idadi kubwa ya Mahakimu wa Mahakama za Mwanzo ambao ni Waswahili, wanaweza kusikiliza, kusoma, kuzungumza na kuandika lugha ya Kiswahili vizuri ingawa mahakimu wamefundishwa na kujifunzia kwa lugha ya Kiingereza katika mfumo mzima wa elimu yao (Wanitzek na Twaib (1996).

Lugha inayotumika katika kuendesha mashauri katika mahakama ya mwanzo ni Kiswahili. Lugha hiyo hujumuisha stadi za kusikiliza, kusema, kusoma na kuandika mwenendo wa shauri. Stadi zote nne ni muhimu katika muktadha wa Mahakama kwa ajili ya maelewano katika kuwasiliana. Lugha ya Kiswahili hutumika isipokuwa tu pale ambapo pande moja hawana uelewa wa lugha ya Kiswahili ndipo mkalimani hutafutwa kwa utaratibu wa kimahakama kama ambavyo tutajadili suala la ukalimani katika sehemu inayofuata. Maswali, ufafanuzi na hojaji chokonoza hufanyika kwa Kiswahili. Kwa kuzingatia hadhira inayokuwepo wakati shauri husika likiendelea, jamii inayofuatilia mwenendo wa shauri, huweza kujifunza lugha ya Kiswahili bila kukusudia katika maeneo yafuatayo:

i. Kunakuwa na mpangilio wa maneno na sentensi kubalifu za lugha ya Kiswahili. Msikizaji hujifunza lugha ya Kiswahili hasa atakapokuwa akisikia jinsi sentensi zinazotumika katika majibizano hayo, maneno yanayoteuliwa kutumika, lugha ya sheria na miktadha yake na misamiati teule ya kisheria kwenye lugha ya Kiswahili.

ii. Kukuza stadi ya kusikiliza na miiko ya uzungumzaji na ushirikishaji wa hadhira hasa pale maswali chokonoza yanapoibuliwa na upande wowote unaoshiriki katika mwenendo wa shauri husika.

\section{Lugha ya Kuandika Mwenendo wa Mashauri}

Lugha ya Kiswahili hutumika katika kuandika mwenendo wa mashauri katika mahakama za Mwanzo. Katika uandishi, Kiswahili kinakua kwa sababu, mwandishi aandikapo mwenendo wa shauri: i. Kuendeleza kanuni bora za uandishi kama vile matumizi ya herufi kubwa, herufi ndogo, mikato na nyinginezo; na

ii. Kuendeleza matumizi sahihi ya aya kwa mawazo mbalimbali yanayoibuka kwenye mwenendo wa shauri husika.

Matumizi ya Kiswahili, kama inavyoonekana katika vipengele (i) na (ii) hapo juu hudhihirika katika nyaraka mbalimbali kama vile katika matini ya hukumu. Matini ya hukumu inagawanyika katika sehemu mbalimbali; sehemu inayohusu utangulizi, dhima ya shauri ambapo mwenendo wote wa shauri husika huonekana hapo, hitimisho ambapo tamko la kama mtuhumiwa ana hatia ama hana hatia hutolewa. Masuala mengine yanayotamkwa katika hatua ya hukumu ni adhabu, jina la mtoa hukumu sahihi na tarehe hukumu inapotolewa na haki ya kukata rufaa. Kuandikwa kwa taarifa zote hizi husaidia kukuza stadi ya lugha husika hususani lugha ya Kiswahili (Carroll, 1995).

\section{Lugha ya Ukalimani katika Mahakama ya Mwanzo}

Mahakama za mwanzo pia hukuza lugha ya Kiswahili katika taaluma ya ukalimani. Ukalimani ni taaluma ya lugha ambapo maudhui yaliyomo katika lugha moja chanzi huelezwa katika lugha nyingine lengwa (Malangwa, 2016). Katika mahakama ya mwanzo, pale ambapo moja wa upande wa washiriki wa shauri hafahamu lugha inayotumika katika mwenendo wa shauri husika, suala la hitaji la ukalimani hujitokeza. Katika mazingira ya Tanzania, kwa kiasi kikubwa, ukalimani mahakamani waweza kufanyika kama mdaiwa hana ufahamu wa lugha mojawapo za jamii na mahakama ikiendesha shauri husika kwa lugha ya Kiswahili. Hapa mkalimani wa lugha ya Kiswahili kutoka lugha ya kijamii au lugha ya kijamii kwenda lugha ya Kiswahili hutumika. Katika mazingira ya mahakama hasa zilizoko mipakani au mijini inapojitokeza shauri linahusisha mdaiwa anayefahamu lugha ya Kiingereza, basi ukalimani utahusisha lugha ya Kiingereza na lugha ya Kiswahili. Ni katika mazingira kama haya ambapo tunaona lugha ya Kiswahili inakua katika stadi ya ukalimani. 


\section{Lugha ya Uandishi wa Machapisho ya Sheria}

Moja wapo ya swali la dodoso katika utafiti huu ilikuwa ni kufahamu iwapo kuna machapisho yoyote ya sheria yaliyoandikwa kwa lugha ya Kiswahili ambayo husaidia kufanya rejea wakati wa kutekeleza majukumu yao. Katika mahakama za Mwanzo nne (4) walisema kwamba kuna vitabu vilivyoandikwa kwa lugha ya Kiswahili na walikiri huwasaidia kuelewa kwa urahisi kuhusu masuala ya sheria yaliyoelezwa kwenye vitabu hivyo. Baadhi ya vitabu hivyo ni:

a. Kitabu cha Sheria kwa Kiswahili juzuu 1. Kitabu hiki kina sheria za ndoa, mirathi, wasia na watoto wa kambo

b. Kitabu cha Sheria za Barabarani. Kitabu hiki kina sheria na kanuni za usalama barabarani (2008)

c. Sheria ya Mwenendo wa Makosa ya Jinai (Sura ya 20)

d. Sheria ya Ardhi ya Vijiji (Sheria Namba 5 ya mwaka 1999)

e. Kanuni ya Adhabu (Sura ya 16)

f. Kiongozi cha Sheria, (Toleo la 5), kimetolewa na kituo cha msaada wa sheria kwa wanawake

g. Sheria ya ndoa na talaka, (2013), kimetolewa na kituo cha msaada wa sheria kwa wanawake

Machapisho mengine yaliyopo kwenye mfululizo huu ni:

a. Makosa ya kujamiiana

b.Ugawaji mirathi kisheria

c. Haki na wajibu kati ya mume na mke

d. Kuishi kinyumba bila ndoa

e. Haki ya mtoto wa kambo kisheria

f. Kutelekeza mimba au mtoto

Tafsiri ya machapisho haya (a-f) kutoka lugha ya Kiingereza kwenda katika lugha ya Kiswahili zinasaidia sana kukuza na kuendeleza Kiswahili.

\section{Lugha ya Matangazo na Mabango katika Ofisi za Mahakama ya Mwanzo}

Mahakama ya mwanzo pia zinakuza lugha ya Kiswahili nchini Tanzania katika matangazo na mabango mbalimbali yanayoonekana katika ofisi za mahakama na za wafawidhi wa mahakama za Mwanzo. Vichwa vya habari vilivyobandikwa ofisini vikionesha orodha za wafawidhi vimeandikwa kwa lugha ya Kiswahili.

\section{Lugha ya Kiswahili katika Uandaaji wa Nyaraka za Kisheria}

Kwa kuangalia maeneo mbalimbali ya wadau wa sheria tunaona kwamba kuna fomu na nyaraka mbalimbali zimetafsiriwa kwa lugha ya Kiswahili. Nyaraka hizi ni kama vile:

a. Fomu mbalimbali za polisi

b. Fomu za kubadilisha majina au viapo vyovyote c. Fomu ya kuitwa shaurini

Kuandaliwa kwa fomu hizi kwa Kiswahili, kumesaidia jamii kufahamu lugha iliyotumika na hivyo kutoa uelewa wa masuala ya sheria na wakati huo huo kukuza Kiswahili.

\section{Uandishi wa Kamusi za Sheria kwa Kiswahili}

Uandishi wa kamusi ya sheria kwa Kiswahili unasaidia sana kukuza na kuenea kwa lugha ya Kiswahili. Hii ni kutokana na vijalizo vinavyotumika na maneno mapya yanayoibuliwa katika kamusi husika. Moja ya kamusi iliyotafsiri sheria kwa Kiswahili ni ile ya Knappert (2001) inayoitwa Law Glossary of Islamic Terms in Swahili. Kamusi hii imetafsiri baadhi ya maneno yanayotumika kwenye sheria za Kiislamu ambapo lugha ya Kiswahili imejipatia utajiri wa istilahi za sheria. Baadhi ya maneno hayo ni kama yafuatavyo:

a. Afifa [n.1-2] a chaste woman; only such a one is suitable for marriage.

b.Ahali [n.9-10] family, clan, but nuclear family, wife and children, parents and siblings.

c. Akibu [n 1-2 pl.ukkabu] follower.

d.Balighi [n.1-2] major, adult. 
e. Bayini [v.t.] show, prove, give evidence of; declare, express.

f. Bulughi [n-9-], being 15 years or older.

g.Chadara [n.5-6] veil: see: sitara.

h.Dhalala [adv.] in vain, in error.

$i$ Dhani [n.9-10] thought, idea, suspicion; personal opinion which is considered fallible even when based on the hadith.

j. Dhuha [n.9] mid-morning; extra prayers which may be performed between 9-10 a.m.

k.Fakiha [n.1-2] law scholar, canon lawyer, theologian, master of jurisprudence.

1. Fakihi [n.1-2 pl. fukaha] law scholar.

m. Fasidi [ a ] irregular, corrupted, spoilt, debauched; invalidated (act).

n. Kiyamu [n.7.] standing position during Islamic prayer.

o.Kongora [n.9-10] (1) bud; (2) the stocks for prisoners.

p.Kusufi [n.9-10] supererogatory prayer, see: nafila.

q. Rataba [n.9-10] 'neck', any object that can be owned.

r. Rihi [n.9-10] wind, flatulence; passing wind makes the state of wudhu invalid; see: nakida.

Tukiangalia mifano katika (a-r) hapa tunaona kwamba, maneno ya kisheria yaliyoko kwenye lugha ya Kiingereza yamepata maana katika Kiswahili. Changamoto yaweza kuwa namna ya usambazaji wa kamusi hizi kuwafikia watumiaji wa Kiswahili ili maneno yaliyotafsiriwa yapate kuzoeleka katika jamii.

\section{NAFASI YA TAASISI ZA SHERIA KATIKA KUKUZA KISWAHILI}

Katika nchi mbalimbali, lugha rasmi imetajwa katika katiba ya nchi au katika sheria mahususi, lakini nchi nyingine hazina sheria juu ya lugha rasmi. Hapa Tanzania, bado vipo vyombo mbalimbali vilivyoundwa kwa utaratibu wa kisheria au bila utaratibu wa kisheria lakini vina nafasi ya kuchangamana na Mahakama katika kuhakikisha kwamba lugha ya Kiswahili inapata kukua na kuenezwa zaidi kimatumizi katika mahakama ya Tanzania. Vyombo hivyo ni kama vifuatavyo:

\section{Taasisi za Elimu ya Sheria Kiwango cha Astashahada na Stashahada}

Katika sehemu hii, utafiti ulilenga kujua kama kuna namna ambayo lugha ya Kiswahili inatumika katika kupata maarifa ya kisheria kwenye taasisi hizi. Taasisi hizo ni kama Chuo cha Uongozi wa Mahakama Lushoto, Chuo Kikuu cha Kumbukumbu ya Sebastiani Kolowa Lushoto na Chuo Kikuu cha Mtakatifu Augustino, Mwanza. Taasisi hizi hutoa mafunzo ya astashahada na stashahada ya sheria. Kiwango cha stashahada kiliteuliwa kwa sababu wahitimu wa kiwango hiki, pamoja na majukumu yao mahala pengine, ndio huajiriwa katika mahakama kuwa wahudumu na makarani; na hivyo wanahusika moja kwa moja katika ushiriki wa mienendo ya mashauri mahakamani na hivyo kuwa na umuhimu katika suala la ukuzaji wa lugha inayotumika mahakamani.

\section{Matumizi ya Lugha ya Kiswahili}

Katika taasisi zote tatu lugha ya kufundishia na kujifunzia ni Kiingereza. Katika programu ya astashahada ya sheria kabla ya kuhitimu kozi yao hutakiwa kufanya mazoezi kwa vitendo kwa majuma manne. Wanafunzi wanaofanyia mazoezi yao kwenye mahakama ya mwanzo hutakiwa kuandika ripoti ya uzoefu wa shughuli za mahakamani kwa mada elekezi ya kuandikia utafiti wao. Kwa majalada ambayo mtafiti aliyaona, inaoneshwa kwamba ripoti hizo huandikwa kwa lugha ya Kiswahili. Mshiriki, yaani hakimu mtarajiwa na karani huandika mwenendo wa mashauri ya jinai na miradi kwa lugha ya Kiswahili kutoka hatua ya mwanzo ya kupokelewa kwa shauri mpaka hatua ya kumalizika kwa shauri. Wanapoandika mwenendo wa mashauri haya, wanaweza kukutana na changamoto za kimsamiati na tafsiri, jambo linalowalazimu kutumia kamusi mahususi. Utatuzi wa changamoto hii, ni hatua kubwa katika kukua kwa lugha ya Kiswahili. Hatua 
hii inasaidia sana kukuza na kuendeleza lugha ya Kiswahili kwa kuwa katika mazoezi kwa vitendo kuna matumizi ya lugha wanayokutana nayo katika maeneo ya mahakama ya mwanzo.

\section{Maigizo katika Mahakama ya Maigizo}

Katika taasisi za elimu mbili (2) kati ya tatu (3) zinazofundisha sheria kwa ngazi ya stashahada ambazo mwandishi alizitembelea kuona kama wana Mahakama ya maigizo na lugha inayotumika katika maigizo haya. Wahusika walieleza kwamba hufanya maigizo ya mwenendo wa mashauri katika mahakama ya Maigizo kwa Kiswahili. Katika mahakama hii kuna pande zote zinazohusika na mwenendo wa shauri kama vile hakimu, wazee wa baraza, wadaawa, waendesha mashtaka kama ni madai au jinai, na mashahidi. Lugha inayotumika katika Mahakama ya maigizo haya ni Kiswahili.

\section{Tume ya Kurekebisha Sheria Tanzania}

Tume ya kurekebisha sheria hufanya tafsiri za sheria mbalimbali kutoka lugha ya Kiingereza kwenda lugha ya Kiswahili. Sheria 62 zimetafsiriwa kutoka lugha ya Kiingereza kwenda lugha ya Kiswahili. Baadhi ya sheria hizo ni kama zifuatazo:

a. Sheria ya Walemavu sura ya 183

b. Sheria ya Mtoto sura ya 13

c. Sheria ya Ukimwi sura ya 431

d. Sheria ya Nafaka na Mazao Mchanganyiko, sura ya 274

e. Sheria ya kuzuia utakatishaji fedha haramu, sura ya 256

f. Sheria ya Mabadiliko ya Katiba ya Mwaka 2012

Mifano (a-f) inaonesha kwamba zipo jitihada zilizokwisha fanyika kutafsiri maandishi ya sheria kutoka Kiingereza kwenda Kiswahili. Jitihada hizi zinachangia ukuaji wa Kiswahili na hivyo tasinia ya sheria kuwa chombo muhimu katika kukua na kuenea kwa Kiswahili.

\section{NAFASI YA TAASISI NYINGINEZO ZINAZOWEZA KUWA NA USHAWISHI KWENYE TASINIA YA SHERIA KATIKA UKUZAJI WA KISWAHILI}

Baada ya kuangalia nafasi ya tasinia ya sheria na vyombo vinavyohusika na sheria katika kukuza lugha ya Kiswahili, ni vizuri kuangalia mchango wa taasisi nyinginezo zisizokuwa za sheria zinavyoweza kutoa msukumo kwenye vyombo vya sheria ili kukuza na kuendeleza Kiswahili. Tunaona vyombo hivi vinginevyo vina mchango mkubwa katika kufanya ushawishi mkubwa kuhusu Mahakama ama taasisi zake zinavyoweza kukuza Kiswahili kwa njia mbalimbali kama inavyofafanuliwa katika sehemu ifuatayo:

\section{Baraza la Kiswahili la Taifa (BAKITA)}

Baraza la Kiswahili la Taifa (BAKITA) lilianzishwa kwa mujibu wa Sheria ya Bunge mwaka 1967, kama chombo cha kitaifa chenye mamlaka wa uendelezaji wa Kiswahili Tanzania. Madhumuni makuu ya chombo hiki yalikuwa kuandaa mazingira ya kukiendeleza Kiswahili, kuhimiza matumizi ya Kiswahili katika maeneo rasmi ya serikali na biashara, kuratibu shughuli zinazohusu Kiswahili na usanifishaji wa Kiswahili. Katika marekebisho ya sheria yaliyofanyika 1983 kwa Sheria ya Bunge Na. 7, BAKITA limepewa uwezo wa kufuatilia na kushirikiana na vyombo vingine katika Jamhuri ya Muungano wa Tanzania ambavyo vinahusika katika kuendeleza lugha ya Kiswahili na kuratibu kazi zao kuhusu matumizi ya lugha ya Kiswahili. Kwa nafasi hii, BAKITA inaweza kutumia mrengo shirikishi kwa kuwa na ubia na mahakama hasa katika kutafsiri nyaraka mbalimbali za kisheria kwa sababu mahakama ni wadau wakubwa wa lugha ya Kiswahili. Pia katika makongamano ambayo BAKITA inayaandaa, inaweza kuwaalika watendaji katika mahakama kama wadau wakuu katika kuendeleza na kukuza Kiswahili kwa njia ya tafsiri na matumizi ya Kiswahili katika muktadha wa Mahakamani na maeneo mengineyo ya sheria.

\section{Taasisi ya Taaluma za Kiswahili (TATAKI)}

Taasisi ya Taaluma za Kiswahili (TATAKI) ni chombo kilicho chini ya Chuo Kikuu cha Dar es Salaam, Tanzania. Taasisi hii ni miongoni mwa 
taasisi kongwe za lugha nchini Tanzania. Madhumuni yake yakiwa kusimamia usanifishaji na maendeleo ya lugha ya Kiswahili. Hadi mnamo mwaka 2009 taasisi hii ilijulikana kwa jina la Taasisi ya Uchunguzi wa Kiswahili (TUKI). Ilianzishwa mnamo mwaka 1930 kama Interteritorial Language Swahili Committee ya nchi za Afrika ya Mashariki, baadaye ikaitwa Kamati ya Kiswahili ya Afrika Mashariki. Mnamo mwaka 1964 kamati ilifanywa kuwa Sehemu ya Chuo Kikuu cha Dar es Salaam. Baadaye ikaitwa Chuo cha Uchunguzi wa Lugha ya Kiswahili. Tangu 1970 kimepewa jina la Taasisi ya Uchunguzi wa Lugha ya Kiswahili. Mwaka 2009, TUKI iliungana na iliyokuwa Idara ya Kiswahili ya Chuo Kikuu cha Dar es Salaam na kuunda chombo kinachoitwa Taasisi ya Taaluma za Kiswahili (TATAKI). Kama ilivyokuwa kwa TUKI, TATAKI pia ilipewa jukumu la kutafiti nyanja zote za lugha ya Kiswahili, fasihi na utamaduni, na kuchapisha matokeo ya utafiti huo. Baada ya kuungana na Idara ya Kiswahili, sasa TATAKI inafundisha isimu, fasihi na historia ya lugha ya Kiswahili katika shahada za awali, mahiri na uzamivu. Kazi yake ilikuwa ni kutafiti na kuchapisha matokeo ya utafiti katika nyanja mbalimbali za lugha, isimu na fasihi ya Kiswahili, TATAKI inasimamia tafsiri na ukalimani. Inafundisha Kiswahili kama lugha ya pili hasa kwa wageni kutoka nje ya ulimwengu wa Waswahili na kuendeleza utungaji wa Kamusi mbalimbali zinazohusiana na Kiswahili.

Hivyo, kwa majukumu haya kama nilivyoyaanisha hapa, TATAKI inaweza kushauri na kushawishi Mahakama ya Tanzania kushirikiana katika kufanya shughuli za ukalimani katika maeneo kadhaa, utungaji wa kamusi za sheria, uandaaji wa vipeperushi vya masuala ya mahakama na tafsiri. Pia, TATAKI inaweza kuanzisha programu za uandishi wa masuala ya sheria kwa Kiswahili. Kwa kufanya hivyo mahakama wanaweza kuwa wadau wakubwa wa kuendeleza lugha ya Kiswahili hapa Tanzania na kwingineko Afrika.

\section{Baraza la Kiswahili Zanzibar (BAKIZA)}

Kama ilivyo kwa BAKITA, Tanzania bara, Baraza la Kiswahili Zanzibar ni chombo kilichopewa mamlaka ya kisheria kukuza na kuendeleza Kiswahili Tanzania Visiwani. Moja kati ya majukumu yake ni kuandaa makongamano ya
Kiswahili ambayo huwakutanisha wataalamu waliobobea kwenye lugha ya kutoka nchi mbali mbali zikiwemo za Afrika Mashariki na kwingineko. Hivyo, tukiangalia majukumu yanayotekelezwa na BAKIZA kama vile kuandaa makongamano yenye malengo ya kukuza lugha adhimu ya Kiswahili, zinaweza pia kuishirikisha Mahakama ya Tanzania kwa kuwakaribisha viongozi wa mahakama kwenye makongamano yao ili kuishawishi mahakama kukitumia Kiswahili katika shughuli mbalimbali za Kimahakama. Hivyo Kiswahili matumizi ya Kiswahili yatakuwa na kuenea katika tasinia ya Mahakama Tanzania Bara na visiwani.

\section{Chama cha Ukuzaji wa Kiswahili Afrika Mashariki (CHAKAMA)}

Chama hiki pamoja na majukumu mengine ya ukuzaji wa lugha ya Kiswahili, kimekuwa mstari wa mbele kuhimiza matumizi ya lugha ya Kiswahili katika maneno mbalimbali ya sheria, lugha ya sheria na mahakama kutumia lugha ya Kiswahili. CHAKAMA kinaweza kwenda mbali zaidi kwa kuwakaribisha watendaji wa Mahakama kwenye makongamano wanayoyaandaa. Watendaji wa mahakama watakapoalikwa kuhudhuria na hata kutoa mada mbalimbali kuhusu Kiswahili na sheria katika makongamano yanayoandaliwa na CHAKAMA, inaweza kutoa ushawishi wa azima ya matumizi ya Kiswahili katika shughuli mbalimbali za Kimahakama katika Mahakama ya ngazi mbalimbali, hivyo kukuza Kiswahili.

\section{Chama cha Ukuzaji Kiswahili Duniani (CHAUKIDU)}

Chama cha Ukuzaji wa Kiswahili Duniani (CHAUKIDU) kilianzishwa kwa malengo ya kuwaunganisha wapenzi, wanataaluma na wasio wanataaluma wa Kiswahili duniani. Madhumuni makuu ya CHAUKIDU ni kuwajumuisha pamoja wakuzaji wa Kiswahili duniani kote kwa lengo la kuchochea kasi ya malengo mahususi yafuatayo:

a. Kukuza Kiswahili katika nyanja zote ambamo lugha hii inatumika au inaweza kutumika na kuongeza ufanisi wa mawasiliano katika ufundishaji na utafiti wa maarifa ya aina zote, uandishi/utangazaji wa habari, uandishi wa vitabu, uchapishaji vitabu na majarida, 
utayarishaji wa safari za mafunzo katika Afrika ya Mashariki na Kati, n.k.

b.Kusambaza habari na matokeo ya utafiti kwa kutumia machapisho mbalimbali na teknolojia ya mtandao wa kompyuta juu ya vipengele mbalimbali vya Kiswahili na masuala yanayohusiana na Kiswahili.

c. Kuwajumuisha wanachama kwa ajili ya kubadilishana mawazo na tajiriba zao katika masuala mbalimbali yanayohusu Kiswahili (kv. uboreshaji wa ufundishaji, utangazaji, utafiti, uandishi, nk.) kwa njia ya mkutano wa kila mwaka, warsha au semina au kongamano maalumu, na hata kwa njia ya mtandao.

d.Kuelimisha umma juu ya umuhimu wa kujivunia, kuendeleza, na kuheshimu Kiswahili.

e.Kushauri ama kushinikiza serikali za nchi ambamo Kiswahili kinazungumzwa kutambua thamani iliyobebwa na maliasili hii ili kuunda sera muafaka za kukiendeleza kadri ya uwezo wake kwa manufaa ya maendeleo ya serikali na watu wake kijamii, kisiasa, kiuchumi, kiutamaduni, kielimu, n.k.

Baada ya kuangalia madhumuni ya CHAUKIDU hapo huu, tunaona kwamba dhumuni (e) huhusika na kushauri serikali katika masuala ya Kiswahili. Hivyo kwa kuzingatia kwamba Mahakama ya Tanzania ni mdau mkubwa wa lugha ya Kiswahili, ushirikiano wa makusudi kati ya CHAUKIDU na Mahakama, hasa kwa njia kuwaalika viongozi wa mahakama kushiriki katika makongamano hayo na kuwasilisha makala yanayohusu Kiswahili na sheria, kutasaidia sana kuenea na kukua lugha ya Kiswahili. Hoja hii inashadidiwa kwa ukweli kwamba katika jamii mahakama ni taasisi inayowafikia watu wengi zaidi hivyo kuwa na matokeo chanya katika kukua na kuenea kwa lugha ya Kiswahili Afrika na duniani kwa ujumla.

\section{CHANGAMOTO}

Baada ya kuangalia namna tasinia ya sheria na taasisi nyinginezo, zinavyochangia kukua na kuenea Kiswahili, sehemu hii tutaangalia changamoto zinazokabili kuenea kwa lugha ya Kiswahili kupitia tasinia ya sheria nchini Tanzania.
Hivyo baadhi ya changamoto hizo ni kama ifuatavyo:

a) Lugha rasmi ya kisheria Tanzania ni Kiingereza. Sheria zote na vitabu vingi vya sheria vimeandikwa katika lugha ya Kiingereza kutokana na mtazamo kwamba Kiswahili kuonekana ni lugha duni isiyoweza kueleza maarifa yaliyomo katika sheria hizo;

b) Kuna hisia miongoni mwa wanasheria na jamii kwa ujumla kwamba tafsiri ya sheria kutoka lugha ya Kiingereza kwenda lugha ya Kiswahili inaweza isiakisi maudhui yaliyomo ndani ya sheria hizo;

c) Watumiaji na wazungumzaji wengi wanahofia kufika sehemu za mahakama hivyo kuifanya lugha ya Kiswahili ingawa inazungumzwa katika maeneo ya Mahakama lakini isipate kusikika na jamii kubwa au mkazo ukawa kusikiliza mashauri na sio ujuzi wa lugha ya Kiswahili;

d) Ukosefu wa rasilimali watu na fedha kwa ajili ya kufanya tafsiri, ukalimani na kuandika sheria kwa lugha ya Kiswahili;

e) Matumizi ya lugha ya Kiingereza katika kufundishia na kujifunzia sheria kwa Watafsiri wa sheria na maafisa katika tasinia ya sheria huleta ugumu wa kutekeleza maarifa hayo kwa lugha ya Kiswahili. Hata mawazo yao yamejengeka katika misingi isiyo bayana kati ya Kiswahili na Kiingereza, hubaki njia panda:

f) Changamoto za kutumia Kiswahili sanifu katika maeneo ya mahakama, hasa za mwanzo kunaweza kuminya mweneo wa lugha ya Kiswahili;

g) Utohoaji wa maneno huwa ni mgumu zaidi hasa inapohusisha maneno ya sheria yaliyopo kwenye lugha ya Kilatini na Kiingereza:

h) Sera ya lugha haiko bayana na haionekani ikijipambanua katika taasisi kama maeneo ya kujifunza na kujifunzia, maeneo rasmi ya serikali: na

i) Athari za ukoloni mamboleo katika lugha ambapo wazungumzaji wa Kiingereza katika 
mahakama za mwanzo huweza kuonekana kama wasomi zaidi kuliko wazungumzaji wa lugha ya Kiswahili. Vipaumbele ni kujua kuzungumza na kuandika vizuri lugha ya Kiingereza.

\section{HITIMISHO NA MAPENDEKEZO}

Makala haya yamejadili njia mbalimbali ambazo Mahakama ya Tanzania inavyochangia katika kukuza na kuendeleza Kiswahili. usikilizaji wa mashauri mahakamani unaweza kusaidia kukua na kuenea kwa lugha ya Kiswahilili hapa Tanzania. Tumeona kwamba, ingawa shughuli zinazofanyika mahakamani ni za kisheria, lakini kwa shughuli mbalimbali za kimahakama kama vile kusikiliza mashauri, kutafsiri sheria na nyaraka mbalimbali, kusoma hukumu katika mahakama za Mwanzo na namna vyombo mbalimbali zilizoundwa kwa kujibu wa sheria zinavyoweza kutoa ushawishi kwa Mahakama kutumia Kiswahili na hivyo kukuza Kiswahili. Kwa kifupi Mahakama inachangia kukuwa kwa Kiswahili kwa njia zifuatazo:

a) Kukuza stadi ya kusoma;

b) Kukuza stadi ya kuandika;

c) Kukuza stadi ya kusikiliza;

d) Kukuza stadi ya kusema;

e) Kukuza taaluma ya ukalimani na tafsiri;

f) Kuongeza vitabu vya rejea; na

g) Kuongeza msamiati katika lugha ya Kiswahili.

Kwa kuwa mahakama za juu na mahakama za mwanzo zinatumia lugha ya Kiingereza, ni wakati muafaka wa kuangalia sheria na sera za lugha ili kwamba lugha ya Kiswahili iwe lugha ya mawasiliano katika mahakama za hakimu mkazi, mahakama kuu na hata mahakama ya rufani nchini Tanzania. Kwa kufanya hivyo itasaidia usambazaji na ukuzaji wa lugha ya Kiswahili, kufahamu yaliyoandikwa katika nakala za hukumu husika katika shauri mahususi ataweza kusoma mwenendo wa mashauri yake katika lugha ya Kiswahili kuliko ilivyo ambapo mwenendo wa shauri huandikwa kwa lugha ya Kiingereza.
Lugha ya Kiswahili iwasemee Waafrika na kuwa nyenzo kuu ya mawasiliano. Viongozi na wadau wakuu wa kuitumia lugha ya Kiswahili ipasavyo kwa sababu viongozi wanaweza kuwa wasimamizi wa matumizi ya Kiswahili katika shughuli rasmi za serikali. Hali hii itakua hata katika sura pana ya kimataifa kama matumizi ya Kiswahili katika mikutano ya Umoja wa Afrika. Lugha ya Kiswahili inaweza kuwa fursa ya ajira.

Wasomi wanaweza kuwa chachu ya mabadiliko ya matumizi ya lugha ya Kiswahili hasa katika matumizi ya lugha ya Kiswahili sanifu vyuoni. Aidha, menejimenti za vyuo zinaweza kurasimisha matumizi ya lugha ya Kiswahili katika vikao mbalimbali vya kimenejimenti.

Katika Mahakama za Tanzania, wateja wengi wanahudumiwa kwa lugha ya Kiswahili. Pia, taarifa nyingi hutolewa kwa lugha ya Kiswahili ingawa lugha inayotumika kufundishia ni Kiingereza. Kwa upande mwingine, kiwango cha wanajamii wanaoweza kuongea lugha za kijamii kinapungua kutokana na watu wengi kuongea lugha ya Kiswahili.

\section{MAREJELEO}

Carroll, J. (1995). The use of interpreters in court. International Journal of Speech, Language and the Law, 2(1), 65-73.

Knappert, J. (2001). Law Glossary of Islamic Terms in Swahili. Mtwara: Benedictine Publications Ndanda.

Malangwa, P. S. (2016). Terminological Challenges and their Impact on the Translation of Specialized Texts: An Analysis of Pharmaceutical Translations from English into Kiswahili. Kioo cha Lugha, 16(1), 126-138.

Mukoyogo, M. C. (1991). Can law be taught in Kiswahili?. Eastern Africa Law Review, 18(2), 227-246.

Rwezaura, B. (1993). Constraining Factors to the Adoption of Kiswahili as a Language of the Law in Tanzania. Journal of African Law, 37(1), 30-45. 
Vlachopoulos, S. (2004). Translating the untranslatable? The impact of cultural constraints on the translation of legal texts. In J. Gibbons, J., Nagarajan, H., Prakasam, V., \& Thirumalesh, K. V., (Eds.) Language in the Law (pp. 100-115). Delhi: Orient Longman.

Wanitzek, U., \& Twaib, F. (1996). The Presentation of claims in matrimonial proceedings in Tanzania: a problem of language and legal culture. $A A P, 47,115-137$. 